\title{
Optimal Power Allocation for the Two-Way Relay Channel with Data Rate Fairness
}

\author{
Mylene Pischella and Didier Le Ruyet, Member, IEEE
}

\begin{abstract}
This letter studies data rate fairness on the twoway relay channel. It analytically determines the optimal power values at both nodes and at the relay, that lead to a maximization of the sum rate under the fairness constraint. Amplify-andForward (AF) and Decode-and-Forward (DF) relaying protocols are considered. For AF, the optimization problem is turned into a single-variable convex optimization problem. For DF, rate balancing between Multiple Access and broadcast phases must be performed prior to setting nodes powers. Both optimized protocols are compared with reference $\mathrm{AF}$ and DF in terms of data rates through numerical simulations.
\end{abstract}

Index Terms-Two-way relay channel, power optimization, fairness, amplify-and-forward, decode-and-forward.

\section{INTRODUCTION}

$\mathbf{T}$ WO-WAY relaying techniques achieve higher spectral efficiency than conventional one-way relaying techniques [1] [2]. On the two-way relay channel with two nodes and one relay, bi-directional transmission requires two time intervals. In the first time interval, both nodes jointly transmit their data to the relay. The channel is then equivalent to a Multiple Access Channel (MAC). In the second time interval, the relay broadcasts the received data to both nodes. Each node removes its own information, called self-interference, and decodes the other node's data. The relaying protocol may be Amplifyand-Forward (AF) [3] [4], Decode-and-Forward (DF) [2], or new protocols making use of recent advances on physical layer network coding [5]. Bi-directional relaying may be an interesting transmission scheme for providing symmetrical data rates, since both nodes may play an identical role in the transmission, provided that adequate power control is performed. It could thus be used for real-time applications like voice or video-conferencing.

In this letter, we investigate fairness in achievable data rates for the SISO two-way relay channel. The objective is to determine the optimal power allocation at both nodes and at the relay that maximizes the sum rate, under a sum power constraint, and under the following fairness constraint: the rate from Node $A$ to Node $B, R_{A B}$, must be equal to the rate from Node $B$ to Node $A, R_{B A}$. To the best of our knowledge, this problem has not been solved yet. AF and DF relaying protocols are studied, in Sections II and III respectively. We provide analytical solutions, and compare the performances with equal power allocation in Section IV.

Manuscript received April 12, 2011. The associate editor coordinating the review of this letter and approving it for publication was N.-D. Dao.

The authors are with the CEDRIC/LAETITIA Laboratory, Conservatoire National des Arts et Métiers, 75003 Paris, France (e-mail: mylene.pischella@cnam.fr).

Digital Object Identifier 10.1109/LCOMM.2011.070711.110789
The following notations are used: $P_{K}$ is the transmit power and $\mathbf{n}_{K}$ is an additive white Gaussian noise with variance $\sigma^{2}$, with $K \in\{A, B, R\}$ corresponding to one of the Nodes or the relay. We consider a narrowband Time Division Duplex system, where the channel coherence time is larger than two time intervals, and where channel reciprocity holds, thanks to accurate calibration of the radio-frequency electronic circuitry [6]. The channel between Node $K \in\{A, B\}$ and the relay is denoted as $h_{K}$. The sum power used in the two time intervals is set to a fixed value, $P_{\text {sum }}=P_{A}+P_{B}+P_{R}$.

\section{AMPLIFY-AND-FORWARD}

In the first time interval, Nodes $A$ and $B$ jointly transmit to the relay. Data are transmitted using a Gaussian codebook. The relay receives $\mathbf{y}_{R}=h_{A} \mathbf{x}_{A}+h_{B} \mathbf{x}_{B}+\mathbf{n}_{R}$. In the second time interval, the relay broadcasts $\gamma \mathbf{y}_{R}$, with an amplification factor $\gamma$ :

$$
\gamma=\sqrt{\frac{P_{R}}{\left|h_{A}\right|^{2} P_{A}+\left|h_{B}\right|^{2} P_{B}+\sigma^{2}}}
$$

Node $A$ receives $\mathbf{y}_{A}=\gamma h_{A}^{2} \mathbf{x}_{A}+\gamma h_{A} h_{B} \mathbf{x}_{B}+\gamma h_{A} \mathbf{n}_{R}+$ $\mathbf{n}_{A}$. The self-interference term, $\gamma h_{A}^{2} \mathbf{x}_{A}$, is removed. Then the Signal to Noise Ratio (SNR) at Node $A$ for the remaining signal $\hat{\mathbf{y}}_{A}=\gamma h_{A} h_{B} \mathbf{x}_{B}+\gamma h_{A} \mathbf{n}_{R}+\mathbf{n}_{A}$ is:

$$
\mathrm{SNR}_{A}=\frac{\gamma^{2}\left|h_{A}\right|^{2}\left|h_{B}\right|^{2} P_{B}}{\gamma^{2}\left|h_{A}\right|^{2} \sigma^{2}+\sigma^{2}}
$$

Similarly, the SNR at Node $B$ is:

$$
\mathrm{SNR}_{B}=\frac{\gamma^{2}\left|h_{B}\right|^{2}\left|h_{A}\right|^{2} P_{A}}{\gamma^{2}\left|h_{B}\right|^{2} \sigma^{2}+\sigma^{2}}
$$

We will now determine the optimal power values, $P_{A}, P_{B}$ and $P_{R}$ that lead to fairness: $\mathrm{SNR}_{A}=\mathrm{SNR}_{B}=\mathrm{SNR}$, and then maximize the SNR. The corresponding sum rate is: $R_{\text {sum }}=$ $\frac{1}{2} \log _{2}\left(1+\mathrm{SNR}_{B}\right)+\frac{1}{2} \log _{2}\left(1+\mathrm{SNR}_{A}\right)=\log _{2}(1+\mathrm{SNR})$.

\section{A. Case $\left|h_{A}\right|^{2}=\left|h_{B}\right|^{2}$}

From eq. (2) and (3), $\mathrm{SNR}_{A}=\mathrm{SNR}_{B}$ if $P_{A}=P_{B}$. Let us denote $|h|^{2}=\left|h_{A}\right|^{2}=\left|h_{B}\right|^{2}$ and $P=P_{A}=P_{B}$. By replacing $P_{R}$ by $P_{\text {sum }}-2 P$, and using eq. (1), we can express the fair SNR as the following function of $P$ :

$$
\mathrm{SNR}=\frac{|h|^{4} P\left(P_{\text {sum }}-2 P\right)}{\sigma^{4}+\sigma^{2}|h|^{2} P_{\text {sum }}}
$$

The SNR is maximized when $g^{\prime}\left(P^{*}\right)=0$, where $g(P)=$ $|h|^{4} P\left(P_{\text {sum }}-2 P\right)$. The optimal value is $P^{*}=\frac{P_{\text {sum }}}{4}$. Consequently, the optimal power allocation achieving fairness when $\left|h_{A}\right|^{2}=\left|h_{B}\right|^{2}$ is: $P_{A}^{*}=P_{B}^{*}=\frac{P_{\text {sum }}}{4}$ and $P_{R}^{*}=\frac{P_{\text {sum }}}{2}$. 
B. Case $\left|h_{A}\right|^{2} \neq\left|h_{B}\right|^{2}$

In that case, $\mathrm{SNR}_{A}=\mathrm{SNR}_{B}$ is equivalent to :

$$
\gamma^{2}=\frac{P_{A}-P_{B}}{\left|h_{B}\right|^{2} P_{B}-\left|h_{A}\right|^{2} P_{A}}
$$

$P_{R}$ can be expressed as a function of $P_{A}$ and $P_{B}$, using (1) and (5):

$$
P_{R}=\frac{\left(P_{A}-P_{B}\right)\left(\left|h_{A}\right|^{2} P_{A}+\left|h_{B}\right|^{2} P_{B}+\sigma^{2}\right)}{\left|h_{B}\right|^{2} P_{B}-\left|h_{A}\right|^{2} P_{A}}
$$

We can notice that there is no solution if $\left|h_{B}\right|^{2} P_{B}=$ $\left|h_{A}\right|^{2} P_{A}$. In all other cases, the fair SNR is equal to:

$$
\mathrm{SNR}=\frac{\left(P_{A}-P_{B}\right)\left|h_{A}\right|^{2}\left|h_{B}\right|^{2}}{\left(\left|h_{B}\right|^{2}-\left|h_{A}\right|^{2}\right) \sigma^{2}}
$$

Two different cases must be distinguished.

1) Case $\left|h_{B}\right|^{2}>\left|h_{A}\right|^{2}$ : The optimization problem corresponding to sum rate maximization is then:

$$
\begin{aligned}
& \max _{\left\{P_{A}, P_{B}\right\}} \log _{2}(1+\mathrm{SNR}) \\
& \text { s.t. }\left|h_{B}\right|^{2} P_{B}-\left|h_{A}\right|^{2} P_{A}>0 \\
& \text { s.t. } P_{A}>P_{B} \\
& \text { s.t. } P_{A}+P_{B}+P_{R}=P_{\text {sum }}
\end{aligned}
$$

where constraints $\left(C_{1}\right)$ and $\left(C_{2}\right)$ are necessary for $\gamma^{2}, P_{R}$ and the SNR to be positive. Using $\left(C_{3}\right)$ and eq. (6), $P_{B}$ can be expressed as functions of $P_{A}$. We obtain:

$$
P_{B}=\frac{\left(\sigma^{2}+\left|h_{A}\right|^{2} P_{\text {sum }}\right) P_{A}}{\sigma^{2}+\left|h_{B}\right|^{2} P_{\text {sum }}+2\left(\left|h_{A}\right|^{2}-\left|h_{B}\right|^{2}\right) P_{A}}
$$

Eq. (7) shows that maximizing the SNR is equivalent to maximizing the difference $\left(P_{A}-P_{B}\right)$ :

$$
\begin{aligned}
P_{A}-P_{B} & =\frac{\left(\left|h_{B}\right|^{2}-\left|h_{A}\right|^{2}\right)\left(P_{\text {sum }} P_{A}-2 P_{A}^{2}\right)}{\sigma^{2}+\left|h_{B}\right|^{2} P_{\text {sum }}+2\left(\left|h_{A}\right|^{2}-\left|h_{B}\right|^{2}\right) P_{A}} \\
& =\left(\left|h_{B}\right|^{2}-\left|h_{A}\right|^{2}\right) f\left(P_{A}\right)
\end{aligned}
$$

Since $\left|h_{B}\right|^{2}>\left|h_{A}\right|^{2}$, this corresponds to maximizing $f\left(P_{A}\right)$.

Constraint $\left(C_{1}\right)$ can also be turned into a function of $P_{A}$, by replacing $P_{B}$ with its expression in (9):

$$
P_{A}<\frac{\sigma^{2}+\left|h_{B}\right|^{2} P_{\text {sum }}}{2\left(\left|h_{B}\right|^{2}-\left|h_{A}\right|^{2}\right)}
$$

Constraint $\left(C_{2}\right)$ becomes $f\left(P_{A}\right)>0$. The denominator of $f\left(P_{A}\right)$ is necessarily positive from eq. (11). Thus, $\left(C_{2}\right)$ is equivalent to $P_{A}<\frac{P_{\text {sum }}}{2}$. This constraint is stronger than (11) since $\frac{P_{\text {sum }}}{2}-\frac{\sigma^{2}+\left|h_{B}\right|^{2} P_{\text {sum }}}{2\left(\left|h_{B}\right|^{2}-\left|h_{A}\right|^{2}\right)}=\frac{-\left(\sigma^{2}+\left|h_{A}\right|^{2} P_{\text {sum }}\right)}{2\left(\left|h_{B}\right|^{2}-\left|h_{A}\right|^{2}\right)}<0$.

The initial optimization problem (8) can consequently be written as the following optimization problem in $P_{A}$ :

$$
\begin{aligned}
& \max _{P_{A}} \frac{P_{\text {sum }} P_{A}-2 P_{A}^{2}}{\sigma^{2}+\left|h_{B}\right|^{2} P_{\text {sum }}+2\left(\left|h_{A}\right|^{2}-\left|h_{B}\right|^{2}\right) P_{A}} \\
& \text { s.t. } P_{A}<\frac{P_{\text {sum }}}{2}
\end{aligned}
$$

Let us study the convexity of function of $f\left(P_{A}\right)=\frac{a P_{A}+b P_{A}^{2}}{c+d P_{A}}$ with $a=P_{\text {sum }}, b=-2, c=\sigma^{2}+\left|h_{B}\right|^{2} P_{\text {sum }}$ and $d=2\left(\left|h_{A}\right|^{2}-\left|h_{B}\right|^{2}\right)$. Its second derivative is:

$$
f^{\prime \prime}\left(P_{A}\right)=-\frac{2 c(a d-b c)}{\left(d P_{A}+c\right)^{3}}
$$

Since $2 c(a d-b c)=4\left(\sigma^{2}+\left|h_{B}\right|^{2} P_{\text {sum }}\right)\left(\sigma^{2}+\left|h_{A}\right|^{2} P_{\text {sum }}\right)>0$, $f^{\prime \prime}$ is of the opposite sign of $g\left(P_{A}\right)=\left(d P_{A}+c\right)^{3} \cdot g\left(P_{A}\right)>0$ if $P_{A}<\frac{\sigma^{2}+\left|h_{B}\right|^{2} P_{\text {sum }}}{2\left(\left|h_{B}\right|^{2}-\left|h_{A}\right|^{2}\right)}$. This is always true since constraint (11) holds. Consequently, in the allowed variation area for $P_{A}, f^{\prime \prime}\left(P_{A}\right)<0$, so function $f$ is concave. The optimization problem (12) is thus convex, and has a unique global optimum. It is equal to the solution of $f^{\prime}\left(P_{A}^{*}\right)=0$ that verifies constraint $\left(C_{1}^{\prime}\right)$. Since $f^{\prime}\left(P_{A}\right)=\frac{b d P_{A}^{2}+2 b c P_{A}+a c}{\left(d P_{A}+c\right)^{2}}$, the potential solutions are the two roots of the numerator. Both roots are real, but only one of them fulfills constraint $\left(C_{1}^{\prime}\right)$. The global optimum of problem (12) is:

$$
\begin{aligned}
& P_{A}^{*}= \\
& \frac{\left(\sigma^{2}+\left|h_{B}\right|^{2} P_{\text {sum }}\right)-\sqrt{\left(\sigma^{2}+\left|h_{B}\right|^{2} P_{\text {sum }}\right)\left(\sigma^{2}+\left|h_{A}\right|^{2} P_{\text {sum }}\right)}}{2\left(\left|h_{B}\right|^{2}-\left|h_{A}\right|^{2}\right)}
\end{aligned}
$$

The optimal values of $P_{B}$, deduced from eq. (9), is:

$P_{B}^{*}=$

$$
\frac{-\left(\sigma^{2}+\left|h_{A}\right|^{2} P_{\text {sum }}\right)+\sqrt{\left(\sigma^{2}+\left|h_{B}\right|^{2} P_{\text {sum }}\right)\left(\sigma^{2}+\left|h_{A}\right|^{2} P_{\text {sum }}\right)}}{2\left(\left|h_{B}\right|^{2}-\left|h_{A}\right|^{2}\right)}
$$

Equations (13) and (14) show that $P_{A}^{*}+P_{B}^{*}=\frac{P_{\text {sum }}}{2}$. This implies that the optimal relay power is always equal to $P_{R}^{*}=$ $\frac{P_{\text {sum }}}{2}$. Thus, the relay gets twice more power than Nodes $A$ and $B$, in order to achieve data rate fairness.

2) Case $\left|h_{A}\right|^{2}>\left|h_{B}\right|^{2}$ : That case can be treated similarly to the previous one, by writting the initial optimization problem (8) as a convex optimization problem in $P_{B}$. The expression of the global optimum, $P_{B}^{*}$, is still (14). Consequently, $P_{A}^{*}$ and $P_{R}^{*}$ are also unchanged.

\section{DECODE-AND-FORWARD}

The rate region of the two-way relay channel, using Decodeand-Forward, has been determined in [2]:

$$
\begin{array}{r}
R_{A B} \leq \frac{1}{2} \min \left\{\log _{2}\left(1+\left|h_{A}\right|^{2} \frac{P_{A}}{\sigma^{2}}\right), \log _{2}\left(1+\left|h_{B}\right|^{2} \frac{P_{R}}{\sigma^{2}}\right)\right\} \\
R_{B A} \leq \frac{1}{2} \min \left\{\log _{2}\left(1+\left|h_{B}\right|^{2} \frac{P_{B}}{\sigma^{2}}\right), \log _{2}\left(1+\left|h_{A}\right|^{2} \frac{P_{R}}{\sigma^{2}}\right)\right\} \\
R_{A B}+R_{B A} \leq \frac{1}{2} \log _{2}\left(1+\left|h_{A}\right|^{2} \frac{P_{A}}{\sigma^{2}}+\left|h_{B}\right|^{2} \frac{P_{B}}{\sigma^{2}}\right)
\end{array}
$$

where the last inequality is due to the joint data rate requirement on the MAC.

In order to maximize the sum rate under the sum power and fairness constraints, $P_{R}$ is set to the minimum value such that the broadcast link does not decrease each point-to-point 


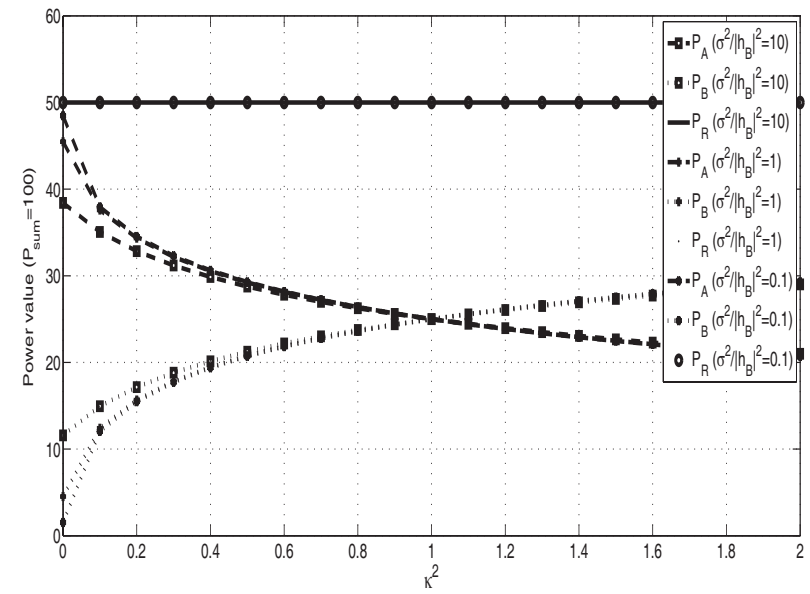

Fig. 1. $P_{A}, P_{B}$ and $P_{R}$ variations as functions of $\kappa^{2}$.

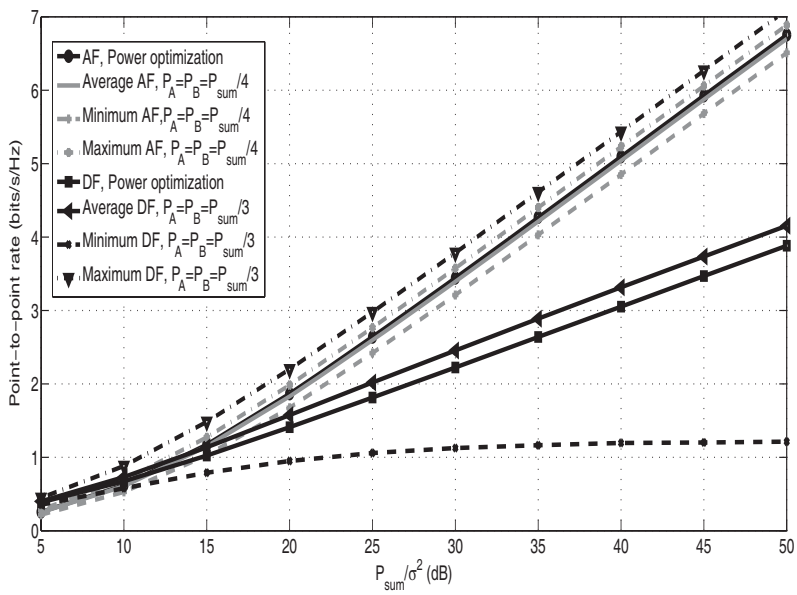

Fig. 2. Point-to-point data rate comparison.

rate. From the first two equations of (15), it is equal to: $P_{R}=$ $\max \left\{\frac{\left|h_{A}\right|^{2}}{\left|h_{B}\right|^{2}} P_{A}, \frac{\left|h_{B}\right|^{2}}{\left|h_{A}\right|^{2}} P_{B}\right\}$.

Then, data rate fairness $R_{A B}=R_{B A}$ can only be achieved if $\left|h_{A}\right|^{2} P_{A}=\left|h_{B}\right|^{2} P_{B}$. By introducing this constraint and $P_{R}$ expression in the sum power, we obtain the optimal power allocation:

$$
\begin{aligned}
& P_{A}^{*}=\frac{P_{\text {sum }}}{D\left|h_{A}\right|^{2}}, P_{B}^{*}=\frac{P_{\text {sum }}}{D\left|h_{B}\right|^{2}}, P_{R}^{*}=\max \left\{P_{A}^{*}, P_{B}^{*}\right\} \\
& \text { with } D=\frac{1}{\left|h_{A}\right|^{2}}+\frac{1}{\left|h_{B}\right|^{2}}+\max \left\{\frac{1}{\left|h_{A}\right|^{2}}, \frac{1}{\left|h_{B}\right|^{2}}\right\}
\end{aligned}
$$

The individual rates are: $R_{A B}=R_{B A}=\frac{1}{2} \log _{2}\left(1+\frac{P_{\text {sum }}}{D \sigma^{2}}\right)$. However, since $\log _{2}\left(1+\frac{P_{\text {sum }}}{D \sigma^{2}}\right) \geq \frac{1}{2} \log _{2}\left(1+2 \frac{P_{\text {sum }}}{D \sigma^{2}}\right)$, the MAC joint data rate constraint always sets the limit for the achievable sum data rate. It is finally equal to :

$$
R_{\text {sum }}=2 R_{A B}=2 R_{B A}=\frac{1}{2} \log _{2}\left(1+2 \frac{P_{\text {sum }}}{D \sigma^{2}}\right)
$$

We can notice that in the particular case when $\left|h_{A}\right|^{2}=\left|h_{B}\right|^{2}$, eq. (16) simplifies to the Equal Power Allocation (EPA) solution: $P_{A}^{*}=P_{B}^{*}=P_{R}^{*}=\frac{P_{\text {sum }}}{3}$.

\section{Simulation Results}

\section{A. Power variations for Amplify-and-Forward}

The variations of the power values depending on $\kappa^{2}=$ $\frac{\left|h_{A}\right|^{2}}{\left|h_{B}\right|^{2}}$, for different levels of $\frac{\sigma^{2}}{\left|h_{B}\right|^{2}}$, are given on Fig. 1. The optimal relay power is always $P_{R}^{*}=\frac{P_{\text {sum }}}{2}$. Consequently, in order to achieve fairness, power must be equally shared between both transmission phases. Besides, in the MAC phase, power is split between nodes so that the node with best channel conditions gets the least power. Power allocation not only depends on the channel gains and on $P_{\text {sum }}$, but also on the noise variance, due to noise amplification at the relay.

\section{B. Point-to-point data rate}

The point-to-point data rates for $\mathrm{AF}$ and $\mathrm{DF}$ with and without power optimization as a function of $\frac{P_{\text {sum }}}{\sigma^{2}}$ is represented on Fig. 2. The reference cases correspond to the optimum values when $\left|h_{A}\right|^{2}=\left|h_{B}\right|^{2}: P_{A}=P_{B}=\frac{P_{\text {sum }}}{4}$ and $P_{R}=\frac{P_{\text {sum }}}{2}$ with AF, and EPA with DF. Fig. 2 also shows the minimum and maximum data rates with these power allocations.

With power optimization, higher data rates are reached with AF than with DF when $\frac{P_{\text {sum }}}{\sigma^{2}}$ exceeds $11 \mathrm{~dB}$. The average data rate is higher with power optimization than in the reference case with AF, but the opposite holds for DF. This is due to the unfairness of DF with EPA. On the contrary, even without power optimization, AF is quite fair, with low differences between the minimum and maximum data rates.

\section{CONCLUSIONS}

This letter has determined the analytical solutions for maximizing the sum rate while achieving fairness on the two-way relay channel, with $\mathrm{AF}$ and DF. Numerical results have shown that fair $\mathrm{AF}$ is more efficient than fair DF, and that fairness also improves the average data rate for $\mathrm{AF}$, compared to reference cases.

\section{REFERENCES}

[1] B. Rankov and A. Wittneben, "Achievable rate regions for the two-way relay channel," in Proc. IEEE ISIT, July 2006.

[2] I. Hammerstrom, M. Kuhn, C. Esli, J. Zhao, A. Wittneben, and G. Bauch, "MIMO two-way relaying with transmit CSI at the relay," in Proc. IEEE SPAWC, June 2007.

[3] J. Ping and S. H. Ting, "Rate performance of AF two-way relaying in low SNR region," IEEE Commun. Lett., vol. 13, pp. 233-235, Apr. 2009.

[4] Y. Zhang, Y. Ma, and R. Tafazolli, "Power allocation for bidirectional AF relaying over Rayleigh fading channels," IEEE Commun. Lett., vol. 14, pp. 145-147, Feb. 2010.

[5] M. P. Wilson and K. Narayanan, "Power allocation strategies and lattice based coding schemes for bi-directional relaying," in Proc. IEEE ISIT, July 2009.

[6] M. Guillaud, D. T. M. Slock, and R. Knopp, "A practical method for wireless channel reciprocity exploitation through relative calibration," in Proc. IEEE ISSPA, Aug.-Sep. 2005. 